\title{
Entropy and entanglement bounds for reduced density matrices of fermionic states
}

\author{
Eric A. Carlen ${ }^{1}$, Elliott H. Lieb ${ }^{2}$ and Robin Reuvers ${ }^{3}$ \\ 1. Department of Mathematics, Hill Center, \\ Rutgers University, 110 Frelinghuysen Road Piscataway NJ 08854-8019 USA \\ 2. Departments of Mathematics and Physics, Jadwin Hall, \\ Princeton University, Washington Road, Princeton, NJ 08544-0001 \\ 3. Department of Physics, Jadwin Hall, \\ Princeton University, Washington Road, Princeton, NJ 08544-0001
}

April 26, 2016

\begin{abstract}
Unlike bosons, fermions always have a non-trivial entanglement. Intuitively, Slater determinantal states should be the least entangled states. To make this intuition precise we investigate entropy and entanglement of fermionic states and prove some extremal and near extremal properties of reduced density matrices of Slater determinantal states.
\end{abstract}

Mathematics subject classification numbers: 81V99, 82B10, $94 \mathrm{~A} 17$

Key Words: Density matrix, Entropy, Partial trace, Entanglement

\section{Introduction}

While bosons are often thought of as more complicated than fermions because of the phenomenon of Bose-Einstein condensation, there is at least one way in which fermions are more complicated, and that is the topic studied here. We investigate the entanglement of fermions caused by the Pauli principle and we seek the minimum possible entropy and

\footnotetext{
${ }^{1}$ Work partially supported by U.S. National Science Foundation grant DMS 1201354.

${ }^{2}$ Work partially supported by U.S. National Science Foundation grant PHY 0965859 and PHY-1265118.

${ }^{3}$ On leave from Department of Mathematical Sciences, University of Copenhagen. Work supported by ERC Grant Agreement Nos. 321029 and 337603.

(C) 2016 by the authors. This paper may be reproduced, in its entirety, for non-commercial purposes.
} 
entanglement. This entanglement is not zero and has been the subject of much discussion in the literature [1], but many questions about the quantification of entanglement forced by statistics remain open. Intuitively, minimal entanglement should occur for (Slater) determinantal states. To make sense of this intuition, quantitative measurements of entanglement are needed.

Our motivation for studying minimal fermionic entanglement and minimal entropy stems from an effort to understand the two-particle density matrix of fermions, which is an important topic for density functional theory and many-body theory. Information about two-particle correlations are potentially useful, and entanglement is one interesting variety of correlation.

It is important to be clear about the definition of entanglement. Several authors define entanglement for fermions as the entanglement relative to a Slater determinantal state. See [1, 10, 16] for reviews and discussion. This difference is sometimes called 'correlation'. In any case, whichever definition one uses, a basic question is to quantify the entanglement of a given multi-particle state and to determine which states minimize various measures of entanglement. The next section will contain precise definitions. While there is a considerable literature on the definition and information theoretic value of various measures of entanglement, it is not our goal here to dwell on this matter. Rather, our goal is to find precise, sharp values for several measures of entanglement and entropy for fermionic states, about which relatively little is known.

Bosons, in contrast to fermions, are not necessarily entangled. The simplest state of $N$ bosons is a condensate, namely the pure state $|\Psi\rangle\langle\Psi|$ where $\Psi$ is a simple product, $\Psi\left(x_{1}, \ldots, x_{N}\right)=\phi\left(x_{1}\right) \cdots \phi\left(x_{N}\right)$ and $\phi$ is normalized. All reduced density matrices are of the same form, i.e., pure products, and all have zero entropy and entanglement. Of course a bosonic state can have arbitrarily high entropy and arbitrarily entangled reduced density matrices, but we are interested in the lowest values. The product states are also important because they form a (necessarily non-orthogonal) basis for bosonic wave functions.

The simplest fermionic wave functions one can think of are Slater determinants. These span the space and an orthonormal basis can be chosen from among them. Our goal is to show that they have the smallest entropy and entanglement - and these minimum values are not zero. From this point of view, determinants are the fermionic analogue of bosonic condensates. Our first theorem considers the mutual information of bipartite fermionic states, which is minimized for 2-particle reduced density matrices of Slater determinants.

Let us make a remark before proceeding. The number $N$ will appear in some theorems and it might be argued that each electron is necessarily entangled with all the electrons in the universe through the Pauli principle, and thus $N \approx \infty$. This is not physically correct, of course. The mathematical solution to this apparent paradox is to realize that a density matrix represents a state on an algebra of observables, and that one must use the lowest dimension possible to accommodate all the observables under consideration in the algebra. One can call this a kind of 'coarse graining'. In our case we imagine that $N$ particles are trapped in a box (or in an isolated atom) and our observables refer only to 
properties inside the box. The Hilbert space for the $N$-body density matrix is then the antisymmetric product $\wedge^{N} \mathcal{H}$, where $\mathcal{H}$ is, e.g., the Hilbert space of one particle (including spin) in the box. All our theorems apply both to finite-dimensional Hilbert spaces and to separable infinite-dimensional ones, but we mention only the finite-dimensional cases for simplicity.

The set-up of the paper is as follows. In Section 2, we focus on mutual information and prove a sharp lower bound on this quantity in Theorem 2.2, which is saturated only for Slater determinants. It is proved using a quantitative subadditivity inequality (Theorem 5.1). We then focus on other measures of entanglement that one might expect are also minimized for Slaters. We prove sharp bounds that are suggested by these minimization problems and formulate a number of conjectures that are related to an old conjecture of Yang concerning the largest possible eigenvalues of fermionic reduced density matrices, Conjecture 3.6. We provide some new information on this in Corollary 3.7. Section 4 analyzes entanglement of formation and squashed entanglement, which are relevant entanglement measures for mixed fermionic states. Section 5 contains the proofs of a number of theorems, including Theorems 2.2 and 5.1 .

Finally, a word on the conventions we use. In many-body physics it is customary for the

$K$-particle reduced matrix to have trace $\left(\begin{array}{l}N \\ K\end{array}\right)$. Standard measures of entanglement involve entropy, however, and to define entropy we require all density matrices $\rho$ to have unit trace $(\operatorname{Tr} \rho=1)$ - as we do here. We also assume that the wedge product of two normalized vectors $\phi$ and $\psi$ has length $\sqrt{2}$, that is, $\phi \wedge \psi:=\phi \otimes \psi-\psi \otimes \phi$. For a wedge product of $K$ vectors, this length is $\sqrt{K !}$. Fermionic projectors, generally denoted by $P$ in this paper, satisfy $P^{2}=P$, so that for example for two particles $P(\phi \otimes \psi)=\phi \wedge \psi / 2$. These conventions should be kept in mind in what follows.

\section{Mutual information}

Let $\rho_{1 \cdots N}$ be a permutation invariant density matrix, as is the case for either bosons or fermions. The $K$-particle reduced density matrix is defined to be

$$
\gamma_{K}=\operatorname{Tr}_{K+1 \cdots N} \rho_{1 \cdots N}
$$

where $\operatorname{Tr}_{K+1 \cdots N}$ denotes the partial trace over the last $N-K$ factors of $\mathcal{H}$.

A permutation invariant state $\rho_{1 \cdots N}$ may be regarded as a bipartite state in $N-1$ different ways, corresponding to the factorizations $\left(\otimes^{K} \mathcal{H}\right) \otimes\left(\otimes^{N-K} \mathcal{H}\right)$ for $K=1, \ldots, N-1$. In what follows, we only consider permutation invariant $N$-particle states.

When $\rho_{1 \ldots N}=|\psi\rangle\langle\psi|$ is a pure state, the entanglement of $|\psi\rangle\langle\psi|$ regarded as a bipartite state on $\left(\otimes^{K} \mathcal{H}\right) \otimes\left(\otimes^{N-K} \mathcal{H}\right)$ is naturally quantified [4] as

$$
S\left(\gamma_{K}\right)=S\left(\gamma_{N-K}\right)
$$


while for mixed states, the issue of quantifying entanglement is more complicated [5]. We return to this later, and focus for now on the entanglement of $N$-particle fermionic states as measured by the quantities in (2.1). It is natural to seek sharp lower bounds on the entropies in (2.1) for $N$-particle fermionic states, which by the concavity of the entropy, will be minimized by pure states.

The only case that is clearly understood is that in which $K=1$ (and by symmetry $K=N-1$ ). Coleman's Theorem (see e.g., [15, Theorem 3.1]), says that the extreme points of the set of all reduced one-particle density matrices of $N$-particle (mixed) fermionic state are the reduced density matrices of $N$-particle Slater determinants, for which all eigenvalues are exactly $1 / N$. That implies that the largest eigenvalue of $\gamma_{1}$ is at most $1 / N$. For any $M$-dimensional density matrix $\rho$ with eigenvalues $\left\{\lambda_{1}, \ldots, \lambda_{M}\right\}$,

$$
S(\rho)=-\sum_{j=1}^{M} \lambda_{j} \ln \left(\lambda_{j}\right) \geq-\sum_{j=1}^{M} \lambda_{j} \ln \|\rho\|_{\infty}=-\ln \|\rho\|_{\infty},
$$

with equality if and only if all positive eigenvalues are equal.

It follows that if $\rho_{1 \ldots N}$ is an $N$-particle fermionic state, then $S\left(\gamma_{1}\right) \geq \ln N$, and there is equality if and only if $\rho_{1 \ldots N}$ is an $N$-particle Slater.

This settles the cases $K=1$ and $K=N-1$, but since we lack an analogue of Coleman's Theorem for other values of $K$, there is no easy route to a lower bound even for $S\left(\gamma_{2}\right)$, although, as we discuss below, it is likely that the lower bound is again given by Slaters. It will be useful to consider the entropies in (2.1) as measures of mutual information.

2.1 DEFINITION. Let $\rho_{1 \ldots l}$ be a density matrix on a Hilbert space $\mathcal{H}_{1} \otimes \cdots \otimes \mathcal{H}_{l}$, and let $\rho_{j}$ be the reduced density matrix on $\mathcal{H}_{j}$. The mutual information in the state $\rho_{1 \ldots l}$ is the quantity

$$
\sum_{j=1}^{l} S\left(\rho_{j}\right)-S\left(\rho_{1 \cdots l}\right)
$$

The difference in (2.2) is well known to be non-negative and zero if and only if $\rho_{1 \cdots N}=$ $\rho_{1} \otimes \cdots \otimes \rho_{l}$. When $\rho_{1 \cdots N}$ is fermionic, this last condition is impossible, and it is natural to seek optimal lower bound on the mutual information for fermionic states.

The entropies in (2.1) can be expressed in terms of mutual informations because if $\rho_{1 \cdots N}$ is a pure fermionic $N$-particle state regarded as a bipartite state on $\left(\otimes^{K} \mathcal{H}\right) \otimes\left(\otimes^{N-K} \mathcal{H}\right)$, then the mutual information is exactly $2 S\left(\gamma_{K}\right)=2 S\left(\gamma_{N-K}\right)$.

2.2 THEOREM (Mutual Information lower bounds). Let $\gamma_{1}, \gamma_{2}$ be the reduced 1, 2-particle density matrices of an $N$-particle fermionic state, respectively. Then

$$
2 S\left(\gamma_{1}\right)-S\left(\gamma_{2}\right) \geq \ln \left(\frac{2}{1-\operatorname{Tr} \gamma_{1}^{2}}\right),
$$

and there is equality if and only if the $N$-particle fermionic state is a pure-state Slater determinant. 
More generally, for an $N$-particle fermionic state $\rho_{1 \ldots N}$

$$
N S\left(\gamma_{1}\right)-S\left(\rho_{1 \ldots N}\right) \geq-\ln e_{N}\left(\gamma_{1}\right)
$$

where $e_{N}\left(\gamma_{1}\right)$ is the $N^{\text {th }}$ elementary symmetric function of the eigenvalues of $\gamma_{1}$, namely, $\sum_{i_{1}<i_{2}<\ldots<i_{N}} \lambda_{i_{1}} \cdots \lambda_{i_{N}}$. This can be expressed in terms of $p_{j}=\operatorname{Tr} \gamma_{1}^{j}$ as

$$
e_{N}=\frac{1}{N !} \operatorname{det}\left[\begin{array}{ccccc}
1 & 1 & 0 & \cdots & \\
p_{2} & 1 & 2 & 0 & \cdots \\
\vdots & & \ddots & \ddots & \\
p_{N-1} & p_{N-2} & \cdots & 1 & N-1 \\
p_{N} & p_{N-1} & \cdots & p_{2} & 1
\end{array}\right]
$$

For example, $e_{3}=\left(1-3 p_{2}+2 p_{3}\right) / 6$.

2.3 Remark. There is equality in (2.3) when $\gamma_{2}$ is is the reduced 2-particle density matrix of an $\mathrm{N}$-particle Slater determinant since, in this case,

$$
S\left(\gamma_{2}\right)=\ln \left(\begin{array}{c}
N \\
2
\end{array}\right), \quad S\left(\gamma_{1}\right)=\ln N, \quad \text { and } \quad \operatorname{Tr} \gamma_{1}^{2}=1 / N .
$$

We now apply this when $\rho_{1 \ldots N}=|\psi\rangle\langle\psi|$ is an $N$-particle fermionic pure state, and seek to estimate the entropies in (2.1). By Jensen's inequality,

$$
e^{-S\left(\gamma_{1}\right)} \leq \operatorname{Tr} \gamma_{1}^{2}
$$

Therefore, (2.3) implies a bound that can be expressed entirely in terms of entropy:

$$
2 S\left(\gamma_{1}\right)-S\left(\gamma_{2}\right) \geq \ln \left(\frac{2}{1-e^{-S\left(\gamma_{1}\right)}}\right) .
$$

If $\gamma_{2}=\rho_{12}$ is pure $S\left(\gamma_{2}\right)=0$, and we have that $2 S\left(\gamma_{1}\right)-\ln \left(2 /\left(1-e^{-S\left(\gamma_{1}\right)}\right)\right) \geq 0$, and this implies that $S\left(\gamma_{1}\right) \geq \ln 2$, which also follows from the fact that the largest eigenvalue of $\gamma_{1}$ is no greater than $1 / 2$, but this shows that even the weakened form (2.7) is sharp.

We now return to the problem of estimating the entropies in (2.1). When $K=1$ or $N-1$ we have seen that Coleman's Theorem provides a complete description of the convex set of reduced one particle density matrices of $N$-particle fermionic states, from which it readily follows that: 1-particle reduced density matrices of Slater determinants minimize the entropy and maximize both the Hilbert-Schmidt norm and the largest eigenvalue.

Less is known about the set of all fermionic reduced 2-body density matrices. Yang's theorem [20] says that no eigenvalue of such a density matrix can exceed $\frac{N}{2}\left(\begin{array}{c}N \\ 2\end{array}\right)^{-1}=1 /(N-$ 1 ), and this is attained for the so-called Yang pairing state (see the start of Section 3 for a definition). This is large compared to $\left(\begin{array}{c}N \\ 2\end{array}\right)^{-1}$, which is the value of every non-zero eigenvalue for a pure Slater determinant. Yang's bound would allow a reduced density matrix with $(N-1)$ eigenvalues close to this size, and hence an entropy of order $\ln (N)$. However, the 2-particle reduced density matrix of the pairing state has a very large entropy, which 
we compute Proposition 3.6, due to a large number of very small eigenvalues, and is not competitive in the search for any entropy minimizer. In contrast, the entropy of a reduced 2-particle density matrix of an $N$-particle Slater state is $\ln \left(\begin{array}{c}N \\ 2\end{array}\right)$, which is of order $2 \ln (N)$. We therefore conjecture:

2.4 Conjecture. The 2-particle reduced density matrix of a Slater determinant minimizes the entropy, that is,

$$
S\left(\gamma_{2}\right) \geq \ln \left(\begin{array}{c}
N \\
2
\end{array}\right)
$$

where $\gamma_{2}$ is the 2-particle density matrix of any $N$-particle fermionic state.

By Jensen's inequality (2.6), this would be implied by the stronger conjecture that 2-particle reduced density matrices of Slater determinants maximize the Hilbert-Schmidt norm:

\subsection{Conjecture.}

$$
\operatorname{Tr}\left[\gamma_{2}^{2}\right] \geq\left(\begin{array}{c}
N \\
2
\end{array}\right)^{-1}
$$

The Yang state also has a squared Hilbert-Schmidt norm of order $N^{-2}$, but it is smaller than the value above. (See Remark 3.5 below.)

A weaker conjecture is:

\subsection{Conjecture.}

$$
S\left(\gamma_{2}\right) \geq 2 \ln N+\mathcal{O}(1)
$$

A strategy to prove this conjecture comes from the proof of Theorem 2.2, which is based on a quantitative subadditivity inequality proved in Theorem 5.1. Suppose now that $N=2 n$, and that $\rho_{1 \cdots N}$ is a fermionic $N$-particle state. Let $\gamma_{2}$ denote its reduced 2 -particle density matrix. The relative entropy of $\rho_{1 \cdots N}$ with respect to the product state $\otimes^{n} \gamma_{2}$ is precisely $n S\left(\gamma_{2}\right)-S\left(\rho_{1 \cdots N}\right)$ and is non-negative on account of the subadditivity of the entropy. Theorem 5.1 below provides a lower bound for this non-negative quantity, which is

$$
S\left(\rho_{1 \cdots N}\right)-\frac{N}{2} S\left(\gamma_{2}\right) \leq 2 \ln \left(\operatorname{Tr}\left[\sqrt{\rho_{1 \cdots N}}\left(\sqrt{\gamma_{2}} \otimes \cdots \otimes \sqrt{\gamma_{2}}\right)\right]\right) .
$$

Since the eigenfunctions of $\sqrt{\rho_{1 \cdots N}}$ are antisymmetric, we may replace $\sqrt{\gamma_{2}} \otimes \cdots \otimes \sqrt{\gamma_{2}}$ in this estimate by $P \sqrt{\gamma_{2}} \otimes \cdots \otimes \sqrt{\gamma_{2}} P$ where $P$ is the projector onto the antisymmetric subspace, and then apply Cauchy-Schwarz to obtain

$$
S\left(\rho_{1 \cdots N}\right)-\frac{N}{2} S\left(\gamma_{2}\right) \leq \ln \left(\operatorname{Tr}\left[P\left(\gamma_{2} \otimes \cdots \otimes \gamma_{2}\right) P\right]\right)
$$

By concavity of the entropy (or convexity of the Hilbert-Schmidt norm), it suffices to consider pure $\rho_{1 \cdots N}$ to prove the conjectures above, in which case

$$
S\left(\gamma_{2}\right) \geq-\frac{2}{N} \ln \left(\operatorname{Tr}\left[P\left(\gamma_{2} \otimes \cdots \otimes \gamma_{2}\right) P\right]\right) .
$$

To study the norm, we prove the following theorem. 
2.7 THEOREM. Let $P$ denote the projector onto $\wedge^{N} \mathcal{H}$ and let $1 \leq K \leq N-1$. Let $M$ be the dimension of the Hilbert space. We define

$$
C_{K}^{M, N}:=\sup _{\substack{\left\|\psi_{1}\right\|=\left\|\psi_{2}\right\|=1 \\ \psi_{1} \in \wedge^{K} \mathcal{H}, \psi_{2} \in \wedge^{N-K \mathcal{H}}}}\left\|P\left(\psi_{1} \otimes \psi_{2}\right)\right\|^{2},
$$

and, using the convention that $\operatorname{Tr}\left(\gamma_{K}^{\Psi}\right)=1$,

$$
\Lambda_{K}^{M, N}:=\sup _{\substack{\|\Psi\|=1 \\ \Psi \in \wedge^{N} \mathcal{H}}} \lambda^{\max }\left(\gamma_{K}^{\Psi}\right)
$$

We then have that $\Lambda_{K}^{M, N}=C_{K}^{M, N}=C_{N-K}^{M, N}=\Lambda_{N-K}^{M, N}$.

Proof. On the one hand, we have for any normalized $\Psi \in \wedge^{N} \mathcal{H}$

$$
\lambda^{\max }\left(\gamma_{K}^{\Psi}\right)=\sup _{\substack{\left\|\psi_{1}\right\|=\left\|\psi_{2}\right\|=1 \\ \psi_{1} \in \wedge^{K} \mathcal{H}, \psi_{2} \in \wedge^{N-K} \mathcal{H}}}\left|\left\langle\Psi, \psi_{1} \otimes \psi_{2}\right\rangle\right|^{2} \leq C_{K}^{M, N} .
$$

The first inequality is obtained by calculating the reduced density matrix from the Schmidt decomposition of $\Psi$, and the second by applying Cauchy-Schwarz and by using that $\Psi=$ $P \Psi$. On the other hand, any normalized $\psi_{1} \in \wedge^{K} \mathcal{H}$ and $\psi_{2} \in \wedge^{N-K} \mathcal{H}$ satisfy

$$
\left\|P\left(\psi_{1} \otimes \psi_{2}\right)\right\|^{2}=\sup _{\substack{\left\|\phi_{1}\right\|=\left\|\phi_{2}\right\|=1 \\ \phi_{1} \in \wedge^{K} \mathcal{H}, \phi_{2} \in \wedge^{N-K \mathcal{H}}}}\left|\left\langle\frac{P\left(\psi_{1} \otimes \psi_{2}\right)}{\left\|P\left(\psi_{1} \otimes \psi_{2}\right)\right\|}, \phi_{1} \otimes \phi_{2}\right\rangle\right|^{2} \leq \Lambda_{K}^{M, N},
$$

where we again applied the Schmidt decomposition to obtain the final inequality.

2.8 COROLLARY. For an $M$-dimensional Hilbert space $\mathcal{H}$, any vectors $\psi_{1} \in \otimes^{K} \mathcal{H}$ and $\psi_{2} \in \otimes^{N-K} \mathcal{H}$ satisfy

$$
\|P(\psi \otimes \tilde{\psi})\|^{2} \leq \Lambda_{K}^{M, N}\left\|\psi_{1}\right\|^{2}\left\|\psi_{2}\right\|^{2},
$$

and any density matrices $\rho_{1}$ on $\otimes^{K} \mathcal{H}$ and $\rho_{2}$ on $\otimes^{N-K} \mathcal{H}$ satisfy

$$
\operatorname{Tr}\left[P\left(\rho_{1} \otimes \rho_{2}\right) P\right] \leq \Lambda_{K}^{M, N} \operatorname{Tr}\left[\rho_{1}\right] \operatorname{Tr}\left[\rho_{2}\right] .
$$

Note that both results can be iterated to obtain further inequalities on composite vectors and density matrices. For $K=2$ and $N=2 n$ and $M=2 m$ even, we have more information from Yang's theorem (see Proposition 3.1): $\Lambda_{2}^{M, N}=(N-1)^{-1}(m-n+1) / m$, and the maximizer is unique.

2.9 COROLLARY. Let $N=2 n$ and $M=2 m$. Let $\psi_{1}, \ldots, \psi_{n} \in \mathcal{H} \wedge \mathcal{H}$ be normalized. We then have

$$
\left\|P\left(\psi_{1} \otimes \cdots \otimes \psi_{n}\right)\right\| \leq \prod_{j=1}^{n} \Lambda_{2}^{M, 2 j}=\prod_{j=1}^{n} \frac{1}{2 j-1} \frac{m-j+1}{m}
$$

with equality if and only if these vectors are all equal and an equal sum of pairs. 
Returning to (2.9), we note that an application of Corollary 2.9 gives the estimate

$$
S\left(\gamma_{2}\right) \geq \ln N+\mathcal{O}(1)
$$

which is a factor 2 off from (2.8). Note that this also follows directly from Yang's theorem and the fact that the entropy is bounded below by $-\ln \left\|\gamma_{2}\right\|_{\infty}$. This estimate is expected to be far from optimal because the 2-particle reduced density matrix of an $N$-particle fermionic state can never be rank 1, which would have be the case to satisfy the bound in Corollary 2.9.

Motivated by the relevance of the Yang state and largest eigenvalues of reduced density matrices, we present some calculations in the next section.

\section{The Yang pairing state}

The Yang pairing state [20] is defined as follows. Let $\mathcal{H}=\mathbb{C}^{M}$ be the one-particle Hilbert space and consider $N \leq M$ particles. Assume that both $M$ and $N$ are even integers, so that we can define integers $m=M / 2$ and $n=N / 2$. We choose an orthonormal basis of $\mathcal{H}$, $u_{i}$ with $1 \leq i \leq M$ and we consider the set of $2 n$-particle Slater determinants $\phi_{\alpha}$ that are composed of $n$ pairs of vectors $\pi_{i}=u_{2 i-1}, u_{2 i}$. There are $m$ such pairs, which are a small fraction of the $\left(\begin{array}{c}2 m \\ 2\end{array}\right)$ pairs with arbitrarily chosen indices. The number of determinants $\phi_{\alpha}$ that we can build from these pairs is $\left(\begin{array}{c}m \\ n\end{array}\right)$. The pairing state (a vector in $\mathcal{H}^{\wedge N}$ ) is given by the equal superposition of these determinants:

$$
\left|\Psi_{M, N}\right\rangle=\left(\begin{array}{l}
m \\
n
\end{array}\right)^{-1 / 2} \sum_{\alpha} \phi_{\alpha}
$$

The eigenvalues of reduced density matrices of the Yang pairing state can give information about its entropic properties, so we will consider these first.

Yang [20] proved the following optimality result for the pairing state.

3.1 PROPOSITION. Let $\gamma_{2}^{\Psi}$ be the 2-particle reduced density matrix of an $N$-particle fermionic state $\Psi \in \wedge^{N} \mathbb{C}^{M}$. If $M=2 m$ and $N=2 n$ are even

$$
\lambda^{\max }\left(\gamma_{2}^{\Psi}\right) \leq \frac{1}{N-1} \frac{m-n+1}{m},
$$

and this is attained if and only if $\Psi$ is a Yang pairing state in some basis.

More generally, let $M=2 m$ if it is even and $M=2 m+1$ if it is odd. Similarly let $N=2 n$ if it is even and $N=2 n+1$ if it is odd. Then, we have that

$$
\lambda^{\max }\left(\gamma_{2}^{\Psi}\right) \leq\left\{\begin{array}{cl}
(N-1)^{-1} & \text { if } N \text { is even } \\
N^{-1} & \text { if } N \text { is odd }
\end{array}\right.
$$

3.2 Remark. Yang's proof of (3.2) uses induction on both $M$ and $N$ and is rather involved. It turns out that the $M \rightarrow \infty$ behaviour follows from a simple argument, which generalizes to odd $N$ and $M$. We now give this simple proof of (3.3). 
Proof. To find the largest possible eigenvalue, we should consider

$$
\sup _{\Psi \in \wedge^{N} \mathcal{H}, f \in \mathcal{H} \wedge \mathcal{H}}\left(f, \gamma_{2}^{\Psi} f\right) .
$$

Given $f \in \mathcal{H} \wedge \mathcal{H}$, a result of Youla [21], and at about the same time Yang [20, states that there are an orthonormal basis $\left\{u_{i}\right\}_{1 \leq i \leq M}$ of $\mathcal{H}$ and positive numbers $\left\{d_{j}\right\}_{1 \leq j \leq m}$ so that

$$
f=\sum_{j=1}^{m} d_{j} \frac{1}{\sqrt{2}} u_{2 j-1} \wedge u_{2 j}
$$

where $\sum_{j}\left(d_{j}\right)^{2}=1$ and the convention for $\wedge$ was mentioned in the introduction.

Let $\boldsymbol{\alpha}$ denote a set of $N$ indices $\left\{\alpha_{1}, \ldots, \alpha_{N}\right\}$ where $1 \leq \alpha_{k} \leq M$, and, if these indices are all different, let $u_{\boldsymbol{\alpha}}$ be given by

$$
u_{\alpha}=u_{\alpha_{1}} \wedge \cdots \wedge u_{\alpha_{N}}
$$

where $\alpha_{1} \leq \cdots \leq \alpha_{N}$. We can expand any state $\Psi$ in Slaters built from $\left\{u_{i}\right\}_{1 \leq i \leq M}$ :

$$
|\Psi\rangle=\sum_{\alpha} c_{\alpha} \frac{1}{\sqrt{N !}} u_{\alpha}
$$

where $c_{\boldsymbol{\alpha}}=0$ if $\boldsymbol{\alpha}$ contains the same index more than once and $\sum_{\boldsymbol{\alpha}}\left|c_{\boldsymbol{\alpha}}\right|^{2}=1$. The 2-particle reduced density matrix is then

$$
\gamma_{2}^{\Psi}=\left(\begin{array}{c}
N \\
2
\end{array}\right)^{-1} \sum_{i<j, i^{\prime}<j^{\prime}}\left(\sum_{\boldsymbol{\beta}} c_{\{i, j\} \cup \boldsymbol{\beta}} \overline{c_{\left\{i^{\prime}, j^{\prime}\right\} \cup \boldsymbol{\beta}}}(-1)^{\sigma(i, j, \boldsymbol{\beta})+\sigma\left(i^{\prime}, j^{\prime}, \boldsymbol{\beta}\right)}\right) \frac{1}{2}\left|u_{i} \wedge u_{j}\right\rangle\left\langle u_{i^{\prime}} \wedge u_{j^{\prime}}\right|
$$

where $\boldsymbol{\beta}$ is a set of $(N-2)$ indices, and $\sigma(i, j, \boldsymbol{\beta})$ is the sign of the permutation that orders $\{i, j\} \cup \boldsymbol{\beta}$.

For $1 \leq j \leq m$, let $p_{j}=\{2 j-1,2 j\}$ denote a pair of indices appearing in (3.4). We have

$$
\begin{aligned}
\left(f, \gamma_{2}^{\Psi} f\right) & =\left(\begin{array}{c}
N \\
2
\end{array}\right)^{-1} \sum_{\boldsymbol{\beta}}\left|\sum_{j} c_{p_{j} \cup \boldsymbol{\beta}}(-1)^{\sigma\left(p_{j}, \boldsymbol{\beta}\right)} d_{j}\right|^{2} \\
& \leq\left(\begin{array}{c}
N \\
2
\end{array}\right)^{-1}\left(\sum_{j} d_{j}^{2}\right) \sum_{\boldsymbol{\beta}, j}\left|c_{p_{j} \cup \boldsymbol{\beta}}\right|^{2} \leq\left(\begin{array}{c}
N \\
2
\end{array}\right)^{-1} n,
\end{aligned}
$$

where we have used Cauchy-Schwarz, $\sum_{j}\left(d_{j}\right)^{2}=1$ and $\sum_{\boldsymbol{\alpha}}\left|c_{\boldsymbol{\alpha}}\right|^{2}=1$, and, also the fact that each $\boldsymbol{\alpha}$ can contain at most $n$ pairs and hence appear at most $n$ times in the sum above.

3.3 Remark. The first line in (3.5) is exact, so it should really give the maximum $\Lambda_{2}^{M, N}$ in the even case. The missing factor compared to (3.6) comes from the fact that there should be no overlap between the indices in $p_{j}$ and $\boldsymbol{\beta}$. This causes the preferred strategy to be to 
spread the weight evenly across the $d$ 's and c's (as the Yang state does). Unfortunately, we have not been able to find a simple argument to prove that this is indeed the best strategy. Since optimization problems for fermions, when written out in coefficients, are difficult because of constraints imposed by the exclusion of repeated indices (such as the constraint $p_{j} \cap \boldsymbol{\beta}=\emptyset$ in (3.5) $)$, it would be good to understand the reason that the optimizers in (3.5) have uniformly distributed coefficients.

The following proposition calculates a number of quantities for a Yang pairing state.

3.4 PROPOSITION. Let $N=2 n$ and $M=2 m$ and let $\gamma_{2}$ be the two-particle reduced density matrix of the $N$-particle pairing state (3.1) built on $\mathcal{H}=\mathbb{C}^{M}$. Its eigenvalues are

$$
\Lambda_{2}^{M, N}=\frac{1}{N-1} \frac{m-n+1}{m}, \quad \lambda_{2}^{M, N}=\frac{1}{N-1} \frac{n-1}{m(m-1)},
$$

where $\Lambda_{2}^{M, N}$ has multiplicity 1 and $\lambda_{2}^{M, N}$ has multiplicity $2 m^{2}-m-1$. Consequently, the entropy is

$$
\begin{aligned}
S\left(\gamma_{2}\right)= & -\frac{m-n+1}{m(2 n-1)} \ln \left[\frac{m-n+1}{m(2 n-1)}\right] \\
& -\frac{(n-1)(2 m+1)}{m(2 n-1)} \ln \left[\frac{(n-1)}{(2 n-1) m(m-1)}\right],
\end{aligned}
$$

and for $p \geq 1$

$$
\operatorname{Tr}\left[\gamma_{2}^{p}\right]=\left(\frac{1}{N-1} \frac{m-n+1}{m}\right)^{p}+\left(2 m^{2}-m-1\right)\left(\frac{1}{N-1} \frac{n-1}{m(m-1)}\right)^{p} .
$$

3.5 Remark. Asymptotically, for $M \gg N \gg 1$, the leading term is $S\left(\gamma_{2}\right) \asymp 2 \ln M$. Thus, $S$ can be much larger than $O(\ln N)$, as it is for a determinant, and can even be infinite. Although the pairing state has a larger eigenvalue (asymptotically $1 / N$ instead of $2 / N^{2}$ ), and potentially a smaller entropy, it has so many small eigenvalues that its entropy can be huge.

Maximizing (3.7) in $M$, we find

$$
\operatorname{Tr}\left[\gamma_{2}^{p}\right] \leq\left\{\begin{array}{ll}
\left(\frac{1}{n(N-1)}\right)^{p-1} & \text { if } p \leq 1+\frac{\ln (N-1)}{\ln (n)} \\
\left(\frac{1}{N-1}\right)^{p} & \text { if } p \geq 1+\frac{\ln (N-1)}{\ln (n)}
\end{array} .\right.
$$

Proof. Let $\gamma$ be the 2-particle reduced density matrix for the pure state $\left|\Psi_{M, N}\right\rangle\left\langle\Psi_{M, N}\right|$, where $\Psi_{M, N}$ is the a pairing state defined in (3.1), normalized so that its trace is $\left(\begin{array}{c}N \\ 2\end{array}\right)$. (This will simplify a number of expressions below.) Note that Yang [20] takes the trace of the 2-particle density matrix to be normalized to $2\left(\begin{array}{c}N \\ 2\end{array}\right)$. 
It is easy to compute the matrix elements of $\gamma$ (with $i<j$ and $k<\ell$ ):

$$
\frac{1}{2}\left(\begin{array}{c}
m \\
n
\end{array}\right)\left\langle u_{i} \wedge u_{j}|\gamma| u_{k} \wedge u_{\ell}\right\rangle= \begin{cases}\left(\begin{array}{c}
m-1 \\
n-1
\end{array}\right) & \text { if } i, j=k, \ell \text { is a pair } \pi_{i} \\
\left(\begin{array}{l}
m-2 \\
n-1
\end{array}\right) & \text { if } i, j \text { and } k, \ell \text { are unequal pairs } \\
\left(\begin{array}{l}
m-2 \\
n-2
\end{array}\right) & \text { if } i, j \text { and } k, \ell \text { are equal and not pairs } \\
0 & \text { otherwise. }\end{cases}
$$

Therefore, the matrix $\gamma$ has the structure

$$
\gamma=\frac{n(m-n)}{m-1}|\chi\rangle\langle\chi|+\frac{n(n-1)}{m(m-1)} P_{\mathcal{H} \wedge \mathcal{H}}
$$

where $\chi=\frac{1}{\sqrt{2 m}} \sum_{j=1}^{m} u_{2 j-1} \wedge u_{2 j}$ is a unit vector, and where $P_{\mathcal{H} \wedge \mathcal{H}}$ is the orthogonal projection in $\mathcal{H} \otimes \mathcal{H}$ onto $\mathcal{H} \wedge \mathcal{H}$. Therefore, $\gamma$ will have two different eigenvalues, with multiplicities $\mu$, as follows:

$$
\frac{n(m-n)}{m-1}+\frac{n(n-1)}{m(m-1)}, \quad \mu=1 ; \quad \frac{n(n-1)}{m(m-1)}, \quad \mu=2 m^{2}-m-1 .
$$

Using either (3.8) or (3.9), one computes that

$$
\operatorname{Tr}(\gamma)=\frac{n(m-n)}{m-1}+\left(2 m^{2}-m\right) \frac{n(n-1)}{m(m-1)}=2 n^{2}-n=\left(\begin{array}{c}
N \\
2
\end{array}\right),
$$

as it must be.

Now letting $\gamma_{2}$ denote the normalized 2-particle reduced density matrix (i.e. with trace 1), we have

$$
\gamma_{2}=\frac{(m-n)}{(2 n-1)(m-1)}|\chi\rangle\langle\chi|+\frac{n-1}{(2 n-1) m(m-1)} P_{\mathcal{H} \wedge \mathcal{H}},
$$

which gives the stated eigenvalues.

One might expect that a similar argument (or the more complete proof by Yang) generalizes to $K$-particle reduced density matrices for $K \geq 3$. However, both proofs hinge on the Yang-Youla description (3.4) of a fermionic bipartite state, which is particularly clear in the proof above.

The Yang-Youla canonical form for vectors in $\Psi \in \wedge^{2} \mathcal{H} \subset \mathcal{H} \otimes \mathcal{H}$ follows easily from the variational characterization of the constituents of the Schmidt decomposition of vectors in $\mathcal{H}_{1} \otimes \mathcal{H}_{2}$. Recall that any such vector has the expansion

$$
\Psi=\sum_{j} \sigma_{j} u_{j} \otimes v_{j}
$$

where $\left\{\sigma_{j}\right\}$ is a non-increasing sequence of non-negative numbers, $\left\{u_{j}\right\}$ is an orthonormal basis of $\mathcal{H}_{1}$ and $\left\{v_{j}\right\}$ is an orthonormal basis of $\mathcal{H}_{2}$. In case $\Psi \in \wedge^{2} \mathcal{H}$, the variational characterization of $\sigma_{1}$ gives

$$
\sigma_{1}=\left|\left\langle\Psi, u_{1} \otimes v_{1}\right\rangle\right| \geq\langle\Psi, u \otimes v\rangle \mid
$$


for all unit vectors $u, v \in \mathcal{H}$. It follows immediately from the antisymmetry of $\Psi$ that $u$ and $v$ are orthogonal, and that $\left|\left\langle\Psi, v_{1} \otimes u_{1}\right\rangle\right|=\left|\left\langle\Psi, u_{1} \otimes v_{1}\right\rangle\right|$. Since $v_{1} \otimes u_{1}$ is orthogonal to $u_{1} \otimes v_{1}$, it is a valid trial vector for $\sigma_{2}$, and thus $\sigma_{2}=\sigma_{1}$. Thus the singular values and the vectors in the Schmidt decomposition come in pairs, and this is precisely the Yang-Youla canonical form (3.4).

Similarly, any $\Psi \in \wedge^{l_{1}+l_{2}} \mathcal{H} \subset \wedge^{l_{1}} \mathcal{H} \otimes \wedge^{l_{2}} \mathcal{H}$ can be written in Schmidt form

$$
\Psi=\sum_{j} \lambda_{j} \psi_{j} \otimes \phi_{j}
$$

where $\psi_{j} \in \wedge^{l_{1}} \mathcal{H}$ and $\phi_{j} \in \wedge^{l_{2}} \mathcal{H}$. Again, $\wedge^{l_{1}} \mathcal{H} \otimes \wedge^{l_{2}} \mathcal{H}$ is much larger than $\wedge^{l_{1}+l_{2}} \mathcal{H}$. The fact that $\Psi$ is antisymmetric, so that $P \Psi=\Psi$, imposes conditions on the Schmidt vectors and Schmidt numbers. While for $l_{1}=l_{2}=1$, it is easy to translate these conditions into the canonical Yang-Youla form, for $l_{1}+l_{2} \geq 3$, the analogue of this canonical form (that would presumably involve vectors of the form $\psi \wedge \phi$ ) is unknown, which is why there is no equivalent of Yang's theorem for $K \geq 3$.

There is, however, a conjecture by Yang [20] regarding this, which remains open after more than fifty years:

3.6 Conjecture (Yang's conjecture). There exist constants $\beta_{3}, \beta_{4}, \ldots$ such that

$$
\Lambda_{K}^{M, N} \leq\left\{\begin{array}{ll}
\frac{(N-K) !}{N !} N^{K / 2} \beta_{K} & \text { if } K \text { is even } \\
\frac{(N-K) !}{N !} N^{(K-1) / 2} \beta_{K} & \text { if } K \text { is odd }
\end{array} .\right.
$$

In (3.11), we assumed that the trace is equal to 1 , rather than the $N ! /(N-K)$ ! that is used in Yang's paper. This formulation of the conjecture is somewhat vague because of the unspecified constants that could be and indeed have to be very large if $K$ is approximately $N / 2$, which will be emphasized below. Theorem 2.7 allows us to be a bit more precise and give lower bounds on these constants, simply by plugging the Yang state as a trial function in the norm problem (2.10), for which it is easy to do computations.

If $M=2 m, N=2 n$ and $K=2 k$, we consider a tensor product of two Yang states built from the same orthonormal basis and find that

$$
\Lambda_{K}^{M, N} \geq\left\|P\left(\Psi_{M, K} \otimes \Psi_{M, N-K}\right)\right\|^{2}=\frac{\left(\begin{array}{l}
n \\
k
\end{array}\right)}{\left(\begin{array}{c}
N \\
K
\end{array}\right)} \frac{\left(\begin{array}{c}
m-n+k \\
k
\end{array}\right)}{\left(\begin{array}{c}
m \\
k
\end{array}\right)}
$$

with $P$ being the antisymmetric projector. As $M \rightarrow \infty$, this tends to

$$
\frac{\left(\begin{array}{l}
n \\
k
\end{array}\right)}{\left(\begin{array}{l}
N \\
K
\end{array}\right)}=\frac{1 \cdot 3 \cdot 5 \cdot \ldots \cdot(K-1)}{(N-1)(N-3)(N-5) \ldots(N-K+1)} .
$$

For $K \approx N / 2$, this gives a largest eigenvalue that seems much higher than Yang's conjecture (3.11). There is no contradiction, however, since the unspecified constants $\beta_{K}$ can be very 
large. It is difficult to say whether there exist fermionic $K$-particle reduced density matrices with higher eigenvalues than (3.12); the key to proving a good upper bound seems to find a canonical Yang-Youla form for $K \geq 3$ as hinted at above.

For completeness, Table 1 below contains lower bounds for odd values of $M, N$ and $K$ that can be found by using Yang states tensored with a fixed element (not present in the pairs from which the pairing state is built) whenever that is necessary.

3.7 COROLLARY. As a corollary of Theorem 2.7, we have lower bounds for the optimal eigenvalues $\Lambda_{K}^{M, N}$ defined in (2.11) that are listed in Table 1 below. Note that the last two rows also give a result for $K=2 k+1$ since $\Lambda_{K}^{M, N}=\Lambda_{N-K}^{M, N}$.

\begin{tabular}{c|c|c|c}
$M$ & $N$ & $K$ & lower bound for $\Lambda_{K}^{M, N}$ \\
\hline $2 m$ or $2 m+1$ & $2 n$ & $2 k$ & $\frac{\left(\begin{array}{l}n \\
k\end{array}\right)}{\left(\begin{array}{l}N \\
K\end{array}\right)} \frac{\left(\begin{array}{c}m-n+k \\
k\end{array}\right)}{\left(\begin{array}{c}m \\
k\end{array}\right)}$ \\
$2 m$ or $2 m+1$ & $2 n$ & $2 k+1$ & $\frac{\left(\begin{array}{c}n-1 \\
k\end{array}\right)}{\left(\begin{array}{l}N \\
K\end{array}\right)} \frac{\left(\begin{array}{c}m-n+k \\
k\end{array}\right)}{\left(\begin{array}{c}m-1 \\
k\end{array}\right)}$ \\
$2 m$ & $2 n+1$ & $2 k$ & $\frac{\left(\begin{array}{l}n \\
k\end{array}\right)}{\left(\begin{array}{c}N \\
K\end{array}\right)} \frac{\left(\begin{array}{c}m-1-n+k \\
k\end{array}\right)}{\left(\begin{array}{c}m-1 \\
k\end{array}\right)}$ \\
$2 m+1$ & $2 n+1$ & $2 k$ & $\frac{\left(\begin{array}{l}n \\
k\end{array}\right)}{\left(\begin{array}{c}N \\
K\end{array}\right)} \frac{\left(\begin{array}{c}m-n+k \\
k\end{array}\right)}{\left(\begin{array}{c}m \\
k\end{array}\right)}$
\end{tabular}

\section{Entanglement for fermionic mixed states}

We now turn to results on bipartite entanglement for fermionic mixed states. There are a range of different entanglement measures that we can consider for mixed fermionic states [5]. In this section we consider two such measures: entanglement of formation and squashed entanglement.

By definition, a bipartite state $\rho_{12}$ state is not entangled if and only if it is separable which means that it is in the closure of states of the form

$$
\rho_{12}=\sum_{k=1}^{n} \nu_{j} \rho_{1}^{j} \otimes \rho_{2}^{j},
$$

where the $\nu_{j}$ are positive and sum to 1 , and each $\rho_{\alpha}^{j}$ is a density matrix on $\mathcal{H}_{\alpha}$. 
The entanglement of formation $\mathrm{E}_{\mathrm{f}}$, introduced by Bennett et al. [4, 5], is defined in terms of the von Neumann entropy $S(\rho)=-\operatorname{Tr}(\rho \log \rho)$ by the formula

$$
\mathrm{E}_{\mathrm{f}}\left(\rho_{12}\right)=\inf \left\{\sum_{j=1}^{n} \lambda_{j} S\left(\operatorname{Tr}_{2} \omega^{j}\right): \rho_{12}=\sum_{j=1}^{n} \lambda_{j} \omega^{j}\right\},
$$

where $\operatorname{Tr}$ and $\operatorname{Tr}_{2}$, respectively, are the traces over the tensor product $\mathcal{H}_{1} \otimes \mathcal{H}_{2}$ and the partial trace over $\mathcal{H}_{2}$ alone. The coefficients $\lambda_{j}$ in the expansion are required to be positive and sum to 1 , and each $\omega^{j}$ is a state on $\mathcal{H}_{1} \otimes \mathcal{H}_{2}$, which, by the concavity of $S$, may be taken to be a pure state without affecting the value of the infimum. Since the two partial traces of a pure state have the same spectrum and hence the same entropies [2], $\mathrm{E}_{\mathrm{f}}\left(\rho_{12}\right)$ is symmetric in 1 and 2 . It is known that $\mathrm{E}_{\mathrm{f}}\left(\rho_{12}\right)=0$ if and only if $\rho_{12}$ is separable; see [6] for a discussion of this result in relation to other measures of entanglement.

Any bipartite fermionic state will be entangled according to this definition. Another definition that is appropriate for fermions is to say that $\rho_{12}$ is fermionic-separable if and only if it is a convex combination of projections onto 2-body Slater determinantal states [1, 13. Otherwise it is fermionic entangled. A number of authors [3, 11, 12, 13, 17, 19] have proposed quantities that measure the degree of fermionic entanglement. One looks for a measure of entanglement that is positive (sometimes called 'faithful') on all entangled states and zero on fermionic-separable states.

We first prove that Slater determinants uniquely minimize the usual 'entanglement of formation', $\mathrm{E}_{\mathrm{f}}$, and, therefore, the excess of $\mathrm{E}_{\mathrm{f}}$ over the Slater value is a faithful measure of fermionic entanglement. This is perhaps the first faithful quantification of fermionic entanglement that uses conventional quantities, like $\mathrm{E}_{\mathrm{f}}$, which have an operational meaning, unlike the 'Slater rank', which is faithful by definition, but which is difficult to compute, is discontinuous, and does not have a clear operational interpretation.

4.1 THEOREM. Let $\rho_{12}$ be a bipartite fermionic state. Then

$$
\mathrm{E}_{\mathrm{f}}\left(\rho_{12}\right) \geq \ln (2),
$$

and there is equality if and only if $\rho_{12}$ is a convex combination of pure-state Slater determinants; i.e., the state is fermionic separable. That is, the quantity

$$
\mathrm{E}_{\mathrm{f}}^{A}\left(\rho_{12}\right):=\mathrm{E}_{\mathrm{f}}\left(\rho_{12}\right)-\ln (2)
$$

is a faithful measure of fermionic entanglement. In particular, if $\rho_{12}$ is the 2-particle reduced density matrix of an $N$-particle fermionic state, then (4.1) is true and equality holds if and only if the state $\rho_{12}$ is fermionic separable.

Proof. As discussed at the start of Section 2, the largest eigenvalue of $\gamma_{1}$ cannot exceed $1 / 2$, and hence any fermionic density matrix $\rho_{12}$ on $\mathcal{H} \otimes \mathcal{H}$ satisfies

$$
S\left(\gamma_{1}\right) \geq \ln 2
$$

and this occurs exactly when $\gamma_{1}$ is the reduced density matrix of a 2-particle Slater determinant. This proves the result since $\mathrm{E}_{\mathrm{f}}$ is a convex combination of such 1-body entropies, and if each is bounded below by $\ln 2$ then so is the convex combination. 
4.2 Remark. Similar statements for pure states were made in [11]. Were we computing the entropy using $\log _{2}$ in place of the natural logarithm, the lower bound would be 1 .

Another faithful measure of entanglement is the squashed entanglement, introduced by Tucci [18] and studied by Christandl and Winter [8]. It is defined by

$$
\mathrm{E}_{\mathrm{sq}}\left(\rho_{12}\right)=\frac{1}{2} \inf _{\rho_{123}}\left\{-S\left(\rho_{123}\right)-S\left(\rho_{3}\right)+S\left(\rho_{13}\right)+S\left(\rho_{23}\right)\right\}
$$

where 3 refers to an additional Hilbert space and $\mathcal{H}=\mathcal{H}_{1} \otimes \mathcal{H}_{2} \otimes \mathcal{H}_{3}$, and $\operatorname{Tr}_{3} \rho_{123}=\rho_{12}$. The infimum is taken over all such extensions of $\rho_{12}$. As a consequence of strong subadditivity [14], $\mathrm{E}_{\mathrm{sq}}\left(\rho_{12}\right) \geq 0$.

The squashed entanglement is a faithful measure of entanglement, meaning that $\mathrm{E}_{\mathrm{sq}}\left(\rho_{12}\right)=0$ if and only if $\rho_{12}$ is separable (in the usual non-fermionic sense) [6]. It is less than or equal to the entanglement of formation, and it is claimed to measure only quantum mechanical correlations.

4.3 Conjecture. Convex combinations of Slater determinantal states uniquely minimize the squashed entanglement, as they do for $\mathrm{E}_{\mathrm{f}}$.

Question: If $\gamma_{2}$ is the 2-particle reduced density matrix of an $N$-particle fermionic state, is $\mathrm{E}_{\mathrm{sq}}\left(\gamma_{2}\right)$ greater than or equal to the squashed entanglement of an $N$-particle Slater determinant? If so, is the difference a faithful measure of fermionic entanglement?

Theorem 4.1 shows this to be the case for entanglement of formation, and the first step in the proof was to compute the $\mathrm{E}_{\mathrm{f}}$ of a two-particle density matrix of a Slater. This number turned out to be independent of $N$. The situation is different for $\mathrm{E}_{\mathrm{sq}}$, for we cannot compute $\mathrm{E}_{\mathrm{sq}}$ for a Slater determinant, but the following theorem, discovered by Christandl, Schuch and Winter [9], definitely shows that there must be an $N$ dependence.

4.4 THEOREM (Squashed entanglement for Slaters). Let $\gamma_{2}$ be the 2-particle reduced density matrix of an $N$-particle Slater determinant. Then

$$
\mathrm{E}_{\mathrm{sq}}\left(\gamma_{2}\right) \leq\left\{\begin{array}{ll}
\ln \frac{N+2}{N} & \text { if } N \text { is even } \\
\frac{1}{2} \ln \frac{N+3}{N-1} & \text { if } N \text { is odd }
\end{array} .\right.
$$

This shows that the squashed entanglement can be much smaller than the entanglement of formation.

4.5 Conjecture. Inequality (4.2) is actually an equality. Moreover, it gives the lowest possible $\mathrm{E}_{\mathrm{sq}}$ among all fermionic 2-particle reduced density matrices.

The fact that $\mathrm{E}_{\mathrm{sq}}$ is so small for a large $N$ fermionic state indicates that the squashed entanglement may already be a good measure of fermionic entanglement, without any further subtraction for large $N$.

For Slaters the entanglement of formation and the squashed entanglement are very different, but the following calculation for the Yang state shows that this need not always be so. 
4.6 THEOREM (Entropy and entanglement of the pairing state). Let $M=2 m$ and $N=2 n$. Let $\gamma_{2}$ be the two-particle reduced density matrix of the $N$-particle pairing state built on $\mathcal{H}=\mathbb{C}^{M}$. Its fermionic entanglement of formation is

$$
\mathrm{E}_{\mathrm{f}}\left(\gamma_{2}\right)-\ln (2)=\frac{(m-n)}{(2 n-1)(m-1)}[\ln (m)-\ln (2)]
$$

and the squashed entanglement is bounded by

$$
\mathrm{E}_{\mathrm{sq}}\left(\gamma_{2}\right) \leq \frac{(m-n)}{(2 n-1)(m-1)} \ln (m)+\left(1-\frac{(m-n)}{(2 n-1)(m-1)}\right) \ln \left(\frac{m+1}{m}\right)
$$

Note that for $M \gg N \gg 1$, the leading term is $\mathrm{E}_{\mathrm{f}}\left(\gamma_{2}\right) \asymp \frac{\ln M}{N}$, and the bound on $\mathrm{E}_{\mathrm{sq}}\left(\gamma_{2}\right)$ is of the same order and is, presumably, close to optimal.

\section{Proofs of Theorems}

While Theorem 2.2 refers to fermionic states, we shall deduce it from the following theorem in which no assumption about statistics is made. It is a quantitative version of subadditivity of the von Neumann entropy, for general bipartite (and $N$-partite) states, which we have not seen before and might be useful in other cases. The method of proof of this theorem also yields quantitative remainder terms for other entropy inequalities - which were discussed in [7.

5.1 THEOREM (Quantitative subadditivity). Let $\rho_{12}$ be a density matrix on a bipartite Hilbert space $\mathcal{H}_{1} \otimes \mathcal{H}_{2}$, and let $\rho_{1}$ and $\rho_{2}$ be its reduced density matrices on $\mathcal{H}_{1}$ and $\mathcal{H}_{2}$, respectively.

$$
S\left(\rho_{12}\right)-S\left(\rho_{1}\right)-S\left(\rho_{2}\right) \leq 2 \ln \left(1-\frac{1}{2} \operatorname{Tr}\left[\sqrt{\rho_{12}}-\sqrt{\rho_{1} \otimes \rho_{2}}\right]^{2}\right) .
$$

In particular, $S\left(\rho_{1}\right)+S\left(\rho_{2}\right)-S\left(\rho_{12}\right) \geq 0$ with equality if and only if $\rho_{12}=\rho_{1} \otimes \rho_{2}$.

More generally, with an obvious notation, if $\rho_{1 \cdots N}$ is a density matrix on $\mathcal{H}_{1} \otimes \cdots \otimes \mathcal{H}_{N}$ then

$$
S\left(\rho_{1 \cdots N}\right)-\sum_{j=1}^{N} S\left(\rho_{j}\right) \leq 2 \ln \left(1-\frac{1}{2} \operatorname{Tr}\left[\sqrt{\rho_{1 \cdots N}}-\sqrt{\rho_{1} \otimes \cdots \otimes \rho_{N}}\right]^{2}\right) .
$$

Proof. Recall the Peierls-Bogoliubov inequality: If $H$ and $A$ are self adjoint operators and Tre $e^{-H}=1$, then

$$
\operatorname{Tr}\left(e^{-H+A}\right) \geq e^{\operatorname{Tr} A e^{-H}} .
$$

To prove (5.1), apply this with

$$
H=-\log \rho_{12} \quad \text { and } \quad A=\frac{1}{2}\left(\log \rho_{1}+\log \rho_{2}-\log \rho_{12}\right) .
$$


Then with $\Delta:=\frac{1}{2}\left(S\left(\rho_{12}\right)-S\left(\rho_{1}\right)-S\left(\rho_{2}\right)\right)$, by the Peierls-Bogoliubov inequality and the Golden-Thompson inequality,

$$
\begin{aligned}
e^{\Delta} & =\exp \left[\operatorname{Tr} \rho_{12} \frac{1}{2}\left(\log \rho_{1}+\log \rho_{2}-\log \rho_{12}\right)\right] \\
& \leq \operatorname{Tr} \exp \left[\frac{1}{2}\left(\log \rho_{12}+\log \left(\rho_{1} \otimes \rho_{2}\right)\right)\right] \\
& \leq \operatorname{Tr} \exp \left[\frac{1}{2} \log \rho_{12}\right] \exp \left[\frac{1}{2} \log \left(\rho_{1} \otimes \rho_{2}\right)\right] \\
& =\operatorname{Tr}\left[\rho_{12}^{1 / 2}\left(\rho_{1} \otimes \rho_{2}\right)^{1 / 2}\right] .
\end{aligned}
$$

Since

$$
\operatorname{Tr}\left[\rho_{12}^{1 / 2}\left(\rho_{1} \otimes \rho_{2}\right)^{1 / 2}\right]=\left(1-\frac{1}{2} \operatorname{Tr}\left[\rho_{12}^{1 / 2}-\left(\rho_{1} \otimes \rho_{2}\right)^{1 / 2}\right]^{2}\right),
$$

this proves (5.1). An obvious adaptation proves (5.2).

Proof of Theorem 2.2. By the hypothesis on $\gamma_{2}$, all of its eigenfunctions with non-zero eigenvalues are antisymmetric, and $\rho_{1}=\rho_{2}=\gamma_{1}$ in Theorem 5.1. Therefore,

$$
\operatorname{Tr}\left(\sqrt{\gamma_{2}} \sqrt{\gamma_{1} \otimes \gamma_{1}}\right)=\operatorname{Tr}\left(\sqrt{\gamma_{2}}\left[P_{\text {fer }} \sqrt{\gamma_{1} \otimes \gamma_{1}} P_{\text {fer }}\right]\right)
$$

where $P_{\text {fer }}$ is the orthogonal projection on the antisymmetric subspace of $\mathcal{H} \otimes \mathcal{H}$, and then by the Cauchy-Schwarz inequality,

$$
\operatorname{Tr}\left(\sqrt{\gamma_{2}} \sqrt{\gamma_{1} \otimes \gamma_{1}}\right)=\operatorname{Tr}\left(P_{\text {fer }} \sqrt{\gamma_{2}} P_{\text {fer }} \sqrt{\gamma_{1} \otimes \gamma_{1}}\right) \leq\left(\operatorname{Tr}\left[P_{\text {fer }} \gamma_{1} \otimes \gamma_{1} P_{\text {fer }}\right]\right)^{1 / 2}
$$

Let $\sum_{j} \lambda_{j}\left|u_{j}\right\rangle\left\langle u_{j}\right|$ denote the spectral decomposition of $\gamma_{1}$. Then

$$
P_{\text {fer }} \gamma_{1} \otimes \gamma_{1} P_{\text {fer }}=\frac{1}{2} \sum_{i<j} \lambda_{i} \lambda_{j}\left|u_{i} \wedge u_{j}\right\rangle\left\langle u_{i} \wedge u_{j}\right|
$$

where $u_{i} \wedge u_{j}=u_{i} \otimes u_{j}-u_{j} \otimes u_{i}$ is a vector of length $\sqrt{2}$, hence the factor of $\frac{1}{2}$. Thus,

$$
\operatorname{Tr}\left[P_{\text {fer }} \gamma_{1} \otimes \gamma_{1} P_{\text {fer }}\right]=\sum_{i<j} \lambda_{i} \lambda_{j}=\frac{1}{2}\left(1-\operatorname{Tr} \gamma_{1}^{2}\right)
$$

Combining this with (5.1), (5.3) and (5.4), we obtain

$$
S\left(\gamma_{2}\right) \leq 2 S\left(\gamma_{1}\right)-\ln \left(\frac{2}{1-\operatorname{Tr} \gamma_{1}^{2}}\right)
$$

The fact that there is equality when $\gamma_{2}$ is the reduced 2-particle density matrix of an $N$-particle Slater has been discussed below the statement of the theorem.

Moreover, whenever there is equality in (5.6), there must be equality in (5.4), in which case for some constant $C, \sqrt{\gamma_{2}}=C P_{\text {fer }} \sqrt{\gamma_{1} \otimes \gamma_{1}} P_{\text {fer }}$, or, what is the same thing by (5.5)

$$
\gamma_{2}=\frac{1}{2} C^{2} \sum_{i<j} \lambda_{i} \lambda_{j}\left|u_{i} \wedge u_{j}\right\rangle\left\langle u_{i} \wedge u_{j}\right| .
$$


Taking the partial trace $\operatorname{Tr}_{2}$ of both sides, we obtain $\gamma_{1}=\frac{1}{2} C^{2} \sum_{j} \lambda_{j}\left(1-\lambda_{j}\right)\left|u_{j}\right\rangle\left\langle u_{j}\right|$, from which we conclude that $\frac{1}{2} C^{2}\left(1-\lambda_{j}\right)=1$ for each $j$. This means that $\gamma_{1}$ is a normalized projection, and that $\gamma_{2}$ is the 2-particle reduced density matrix of a pure Slater determinant.

The inequality (2.4) follows from (5.2) in the same way that (2.3) follows from (5.1). The equation (2.5) for $e_{N}$ in terms of power sums (using $p_{1}=\operatorname{Tr} \gamma_{1}=1$ ) is well known.

Proof of Theorem 4.4. Let $\Psi$ be an $N$-particle Slater determinant. We choose $\rho_{123}$ to be the $K$-particle reduced density matrix with $K \geq 2$. Thus, $\mathcal{H}_{3}$ is the $(K-2)$-particle fermionic space, which has dimension $\left(\begin{array}{c}N \\ K-2\end{array}\right)$. We compute as follows:

$$
S\left(\rho_{123}\right)=\ln \left(\begin{array}{l}
N \\
K
\end{array}\right), S\left(\rho_{3}\right)=\ln \left(\begin{array}{c}
N \\
K-2
\end{array}\right), S\left(\rho_{13}\right)=S\left(\rho_{23}\right)=\ln \left(\begin{array}{c}
N \\
K-1
\end{array}\right) .
$$

Thus,

$$
-S\left(\rho_{123}\right)-S\left(\rho_{3}\right)+S\left(\rho_{13}\right)+S\left(\rho_{23}\right)=\ln [K /(N-K+1)]+\ln [(N-K+2) /(K-1)],
$$

and the theorem is proved by choosing $K=(N+2) / 2$ for $N$ even and $K=(N+1) / 2$ (or $K=(N+3) / 2)$ for $N$ odd.

Proof of Theorem 4.6. Proposition 3.4 and its proof can be used to compute $\mathrm{E}_{\mathrm{f}}\left(\gamma_{2}\right)$ and to estimate $\mathrm{E}_{\mathrm{sq}}\left(\gamma_{2}\right)$. Let $\alpha$ denote the completely antisymmetric state on $\mathcal{H} \otimes \mathcal{H}$; i.e., $\alpha=(m(2 m-1))^{-1} P_{\mathcal{H} \wedge \mathcal{H}}$. Then we may write (3.10) as

$$
\gamma_{2}=\frac{(m-n)}{(2 n-1)(m-1)}|\chi\rangle\langle\chi|+\left(1-\frac{(m-n)}{(2 n-1)(m-1)}\right) \alpha .
$$

In any decomposition $\gamma_{2}=\sum_{j=1}^{n} \lambda_{j} \omega^{j}$, one of the $\omega_{j}$ must be $|\chi\rangle\langle\chi|$ and the corresponding $\lambda_{k}$ must be at least $(m-n) /(2 n-1)(m-1)$. Since $S\left(\operatorname{Tr}_{1}|\chi\rangle\langle\chi|\right)=\ln m$, it follows from these computations and Theorem 4.1 that

$$
\mathrm{E}_{\mathrm{f}}\left(\gamma_{2}\right)-\ln (2)=\frac{(m-n)}{(2 n-1)(m-1)}[\ln (m)-\ln (2)] .
$$

Since $\mathrm{E}_{\mathrm{sq}}(|\chi\rangle\langle\chi|)=\ln (m)$ it follows from the convexity of squashed entanglement [8] and Theorem 4.4 that

$$
\mathrm{E}_{\mathrm{sq}}\left(\gamma_{2}\right) \leq \frac{(m-n)}{(2 n-1)(m-1)} \ln (m)+\left(1-\frac{(m-n)}{(2 n-1)(m-1)}\right) \ln \left(\frac{m+1}{m}\right) .
$$

Note that for $M \gg N \gg 1$, the leading term is $\mathrm{E}_{\mathrm{f}}\left(\gamma_{2}\right) \asymp \frac{\ln M}{N}$, and the upper bound on $\mathrm{E}_{\mathrm{sq}}\left(\gamma_{2}\right)$ is of the same order and is, presumably, close to optimal.

Acknowledgements: We thank U. Marzolino and J. Schliemann for helpful correspondence. We thank the U.S. National Science Foundation for support by grants DMS 1501007. (E.A.C.) and PHY 0965859 and PHY-1265118 (E.H.L.) and the European Research Council for support by ERC Grant Agreement Nos. 321029 and 337603 (R.R.). 


\section{References}

[1] L. Amico, R. Favio, A. Osterloh and V. Vedral, Entanglement in many-body systems, Rev. Mod. Phys. 80, 517-576 (2008)

[2] H. Araki and E. H. Lieb, Entropy Inequalities, Commun. Math. Phys. 18, 160-170 (1970).

[3] M. Bañuls, J. I. Cirac and M. M. Wolf, Entanglement in fermionic systems, Phys. Rev. A 76, 022311 (2007).

[4] C.H. Bennett, G. Brassard, S. Popescu, B. Schumacher, J.A. Smolin, W.K. Wootters, Purification of noisy entanglement and faithful teleportation via noisy channels, Phys. Rev. Lett., 76, no. 5, 1996, pp. 722-725, Erratum: Phys. Rev. Lett., 78, no. 10, 1997, p. 2031.

[5] C.H. Bennett, D.P. DiVincenzo, J.A. Smolin, W.K. Wootters, Mixed state entanglement and quantum error correction Phys. Rev. A, 54, no. 5, 1996, pp. 3824-3851.

[6] F. Brandao, M. Christandl, and J. Yard, Faithful squashed entanglement Commun. Math. Phys. 306, 2011, pp 805

[7] E. A. Carlen and E. H. Lieb, Remainder Terms for Entropy inequalities, Jour. Math. Phys 55, $042201(2014)$

[8] M. Christandl and A. Winter, Squashed entanglement - An additive entanglement measure. J. Math. Phys.,45, 2004, pp 829-840.

[9] M. Christandl, N. Schuch and A. Winter, Entanglement of the antisymmetric state, Commun. Math. Phys. 311, 397-422 (2012).

[10] K. Eckert, J. Schliemann, D. Bruß and M. Lewenstein. Quantum Correlations in Systems of Indistinguishable Particles, Annals of Physics 299, 88127 (2002).

[11] G. Ghiradi and L Marinatto, General criterion for the entanglement of two indistinguishable particles, Phys. Rev. A 70, 012109

[12] F. Iemini and R. O. Vianna, Computable measures for the entanglement of indistinguishable particles, Phys. Rev. A 87, 022327 (2013).

[13] C. V. Kraus, M. M. Wolf, J. I. Cirac, and G. Giedke, Pairing in fermionic systems: A quantum-information perspective, Phys. Rev. A 79, 012306 (2009).

[14] E. H. Lieb and M. B. Ruskai, Proof of the Strong Subadditivity of Quantum-Mechanical Entropy,i J. Math. Phys. 14, 1938-1941 (1973).

[15] E. H. Lieb and R. Seiringer The Stability of Matter in Quantum Mechanics, Cambridge University Press, Cambridge (2010).

[16] A. Peres, Quantum Theory: Concepts and Methods, Kluwer Academic, Dordrecht, (1993). 
[17] J. Schliemann, J. I.Cirac, M. Kuś, M. Lewenstein and D. Loss, Quantum correlations in two-fermion systems, Phys. Rev. A 64, 022303 (2001).

[18] R. Tucci, Entanglement of distillation and conditional mutual information, eprint quant-ph/0202144, (2002).

[19] X. Wang and B. C. Sanders, Canonical entanglement for two indistinguishable particles, J. Phys. A: Math. Gen. 38, L67-L72 (2005).

[20] C. N. Yang, Concept of off-diagonal long-range order and the quantum phases of liquid He and of superconductors, Reviews of Modern Physics 34, 694-704 (1962).

[21] D. C. Youla, A normal form for a matrix under the unitary congruence group, Canad. J. Math. 13, 694-704, (1961). 